\title{
Prognostic indicators in adults hospitalized with falciparum malaria in Western Thailand
}

\author{
Paul N Newton ${ }^{1,3^{*}}$, Kasia Stepniewska ${ }^{2,3}$, Arjen Dondorp $^{2,3}$, Kamolrat Silamut ${ }^{2}$, Wirongrong Chierakul ${ }^{2}$, \\ Sanjeev Krishna ${ }^{4}$, Timothy ME Davis ${ }^{5}$, Yupin Suputtamongkol ${ }^{6}$, Brian Angus $^{3}$, Sasithon Pukrittayakamee ${ }^{2}$, \\ Ronnatrai Ruangveerayuth ${ }^{7}$, Josh Hanson ${ }^{2,8}$, Nicholas PJ Day ${ }^{2,3}$ and Nicholas J White ${ }^{2,3}$
}

\begin{abstract}
Background: Severe malaria remains a major cause of death and morbidity amongst adults in the Asiatic tropics. Methods: A retrospective analysis of the clinical and laboratory data of 988 adult patients, hospitalized with Plasmodium falciparum malaria and prospectively recruited to malaria studies in western Thailand between 1986 and 2002, was performed to assess the factors associated with a fatal outcome. Different severity scores and classifications for defining severe malaria were compared and, using multiple logistic regression, simple models for predicting mortality developed.

Results: The proportion of patients fulfilling the WHO 2000 definition of severe malaria was 78.1\%, and their mortality was $10 \%$. Mortality in patients given parenteral artesunate or artemether $(16 / 317,5 \%)$ was lower than in those given parenteral quinine (59/442, 13\%) (P<0.001). Models using parameter sets based on WHO 1990, 2000 and Adapted AQ criteria plus blood smear parasite-stage assessment gave the best mortality prediction. A malaria prognostic index (MPI), derived from the dataset using five clinical or laboratory variables gave similar prognostic accuracy.

Conclusions: The mortality of severe malaria in adults has fallen and the switch from quinine to artesunate has probably been an important contributor. Prognostic indices based on WHO 2000 definitions, and other simpler indices based on fewer variables, provide clinically useful predictions of outcome in Asian adults with severe malaria.
\end{abstract}

Keywords: Malaria, Mortality, Thailand, Plasmodium falciparum, Prognosis

\section{Background}

Plasmodium falciparum malaria still kills $\sim 0.7$ million people each year. The majority of those who die are children in sub-Saharan Africa, but 43,000 patients still die each year in the Asia-Pacific [1]. Admission clinical features that predict death have been defined in African children [2-6]. For Asian adults the relationships between mortality and a wide range of individual variables have been tested, including parasitaemia and parasite-stage distribution and intraleucocytic malaria pigment on blood smears, renal failure, hypoglycaemia, cerebral malaria, acidosis and older age [7-14] (Additional file 1, Tables 1 and 2). However, there have been few assessments

\footnotetext{
* Correspondence: paul@tropmedres.ac

'Lao-Oxford-Mahosot Hospital-Wellcome Trust Research Unit, Microbiology

Laboratory, Mahosot Hospital, Vientiane, Lao PDR

${ }^{3}$ Centre for Tropical Medicine, Churchill Hospital, Nuffield Department of

Medicine, University of Oxford, Oxford OX3 7LJ, UK

Full list of author information is available at the end of the article
}

of which combinations of prognostic factors are the best predictors of mortality. Although a single definition may not be appropriate everywhere, it is important to attempt to define severe malaria to aid clinicians in recognizing those at risk of death and in need of parenteral therapy. This will inform decisions on whether transfer to a higher level of care, if available, is appropriate, facilitate comparison between datasets, longitudinal epidemiological surveillance, and robust case definitions in evaluation of interventions $[11,15]$.

Several definitions, classifications and severity scores have been proposed. The World Health Organization (WHO) has produced three guidelines which include definitions of severe malaria (Additional file 1, Tables 1 and 2) [16-18]. A slightly stricter definition of severe malaria ('AQ') was developed for a clinical trial in Vietnam [19] and adapted (with the addition of plasma lactate and serum bicarbonate measurements) for a trial in Thailand 
Table 1 Clinical variables included in the ten sets, used in the logistic regression analyses

\begin{tabular}{|c|c|c|c|c|c|c|c|c|c|c|}
\hline Variable & $\begin{array}{l}\text { Bed } \\
\text { side }\end{array}$ & $\begin{array}{l}\text { Bedside and } \\
\text { simple lab }\end{array}$ & $\begin{array}{l}\text { WHO } \\
1990 \\
\end{array}$ & $\begin{array}{l}\text { WHO } \\
2000 \\
\end{array}$ & $\begin{array}{l}\text { Adapted } \\
\text { AQ }\end{array}$ & $\begin{array}{l}\text { Adapted AQ } \\
\text { plus late stages }\end{array}$ & BCAM & RCAM & MSA & MPI \\
\hline Seizures before admission & $x$ & $x$ & $x$ & $x$ & - & - & - & - & - & - \\
\hline Clinical anaemia & $x$ & $x$ & - & - & - & - & - & - & - & - \\
\hline Clinical jaundice & $x$ & $x$ & $x$ & $x$ & - & - & - & - & - & - \\
\hline Bleeding & - & - & $x$ & $x$ & - & - & - & - & - & - \\
\hline Blackwater fever & - & - & $x$ & $x$ & - & - & - & - & - & - \\
\hline Temperature & $x$ & $x$ & $x$ & - & - & - & - & - & - & - \\
\hline Pulse & $x$ & $x$ & - & - & - & - & - & - & - & - \\
\hline Respiratory rate & $x$ & $x$ & - & $x$ & - & - & - & $x$ & - & - \\
\hline $\begin{array}{l}\text { Respiratory distress, requiring } \\
\text { mechanical ventilation }\end{array}$ & - & - & - & - & - & - & - & - & $x$ & - \\
\hline Liver palpable & $x$ & $x$ & - & - & - & - & - & - & - & - \\
\hline GCS Total & $x$ & $x$ & $x$ & $x$ & $x$ & $x$ & $x$ & $x$ & $x$ & $x$ \\
\hline Pulmonary oedema & - & - & $x$ & $x$ & - & - & - & - & - & - \\
\hline
\end{tabular}

$\mathrm{X}=$ included, - = not included.

BCAM score uses serum bicarbonate as a marker of acidosis with cut-off values of $\geq 24 \mathrm{mmol} / \mathrm{L}$ (score $=0$ ) for normal, $\geq 15-<24 \mathrm{mmol} / \mathrm{L}$ (score $=1$ ) for deranged, and $<15 \mathrm{mmol} / \mathrm{L}$ (score $=2$ ) for very deranged along with thresholds for coma of GCS $\leq 14$ and GCS $\leq 10$ [23]. The acidosis and coma scores were summed to give the BCAM score, ranging from $0-4$.

RCAM score uses respiratory rate as a surrogate marker for acidosis with cut offs of $<20$ breaths/min (score $=0$ ) for normal, $20-39$ breaths/min (score $=1$ ) for deranged, and $\geq 40$ breaths/min (score $=2$ ) for very deranged.

The MSA score was defined as sum of 1 (severe anaemia [haemoglobin, $<5 \mathrm{~g} / \mathrm{dL}$ ]) +2 (acute renal failure [serum creatinine, $>3 \mathrm{mg} / \mathrm{dL}$ or $250 \mu \mathrm{mol} / \mathrm{L}$ ]) +3 (respiratory distress, requiring mechanical ventilation) +4 (cerebral malaria [GCS $<11])$, in which each variable was scored as 0 or 1 , depending on its absence or presence, respectively [22].

Table 2 Laboratory variables included in the ten sets, used in the logistic regression analyses

\begin{tabular}{|c|c|c|c|c|c|c|c|c|c|c|}
\hline Blood test variable & $\begin{array}{l}\text { Bed } \\
\text { side }\end{array}$ & $\begin{array}{l}\text { Bedside and } \\
\text { simple lab }\end{array}$ & $\begin{array}{l}\text { WHO } \\
1990 \\
\end{array}$ & $\begin{array}{l}\text { WHO } \\
2000 \\
\end{array}$ & $\begin{array}{c}\text { Adapted } \\
\text { AQ } \\
\end{array}$ & $\begin{array}{c}\text { Adapted AQ } \\
\text { plus late stages }\end{array}$ & BCAM & RCAM & MSA & MPI \\
\hline Admission parasitaemia & - & $x$ & $x$ & $x$ & $x$ & $x$ & - & - & - & $x$ \\
\hline Pigmented stages & - & $x$ & - & - & - & $x$ & - & - & - & $x$ \\
\hline Modal Stage & - & $x$ & - & - & - & - & - & - & - & - \\
\hline Haematocrit & - & $x$ & $x$ & $x$ & $x$ & $x$ & - & - & - & - \\
\hline Haemoglobin & - & - & - & - & - & - & - & - & $x$ & - \\
\hline White cell count & - & - & - & - & - & - & - & - & - & - \\
\hline Potassium $^{\mathrm{S}}$ & - & - & - & - & - & - & - & - & - & - \\
\hline Creatinine $^{s}$ & - & - & $x$ & $x$ & $x$ & $x$ & - & - & $x$ & - \\
\hline Urea $^{\mathrm{s}}$ & - & - & - & - & - & - & - & - & - & - \\
\hline Urea:creatinine ratio $^{\mathrm{s}}$ & - & - & - & - & - & - & - & - & - & - \\
\hline Total bilirubin $^{\mathrm{S}}$ & - & - & $x$ & - & $x$ & $x$ & - & - & - & $x$ \\
\hline Direct bilirubin ${ }^{\mathrm{s}}$ & - & - & - & - & - & - & - & - & - & - \\
\hline Alkaline phosphatase ${ }^{S}$ & - & - & - & - & - & - & - & - & - & - \\
\hline $\mathrm{AST}^{\mathrm{S}}$ & - & - & - & - & - & - & - & - & - & - \\
\hline$A L T^{S}$ & - & - & - & - & - & - & - & - & - & - \\
\hline Albumin $^{\mathrm{S}}$ & - & - & - & - & - & - & - & - & - & - \\
\hline Bicarbonate $^{\mathrm{S}}$ & - & - & $x$ & $x$ & $x$ & $x$ & $x$ & - & - & - \\
\hline Glucose $^{P}$ & - & - & $x$ & $x$ & $x$ & $x$ & - & - & - & - \\
\hline Lactate $^{P}$ & - & - & - & $x$ & $x$ & $x$ & - & - & - & $x$ \\
\hline
\end{tabular}

$\mathrm{X}=$ included,$-=$ not included. ${ }^{\mathrm{S}}=$ serum ${ }^{\mathrm{P}}=$ plasma. 
[20] and the subsequent multicentre SEAQUAMAT trial which enrolled 1461 Asian patients [21]. The malaria severity assessment score (MSA), based on haemoglobin, serum creatinine, requirement for mechanical ventilation and Glasgow coma score (GCS) was developed in central India [22].

Recently, in order to simplify and, therefore, broaden usage, the coma acidosis malaria (CAM) score was developed [23], based on data from Asian adults with severe malaria [21]. A score of $<2 / 5$, when tested with data from different studies in Vietnamese and Bangladeshi adults, had a positive predictive value (PPV) for survival of 94-95\%, suggesting that these patients could be cared for without admission to an intensive care unit (ICU).

The clinical and laboratory features of adults with falciparum malaria recruited prospectively to hospitalbased studies on the western border of Thailand between 1986-2002, conducted by the Mahidol University-Oxford Tropical Medicine Research Unit and colleagues, were analysed. The specificity and sensitivity of the different severe malaria definitions and scoring systems were examined and simple models to identify adults at risk of death built.

\section{Methods}

\section{Study sites, years and studies}

Data from 988 adult ( $\geq 15$ years) patients with asexual forms of $P$. falciparum present on peripheral blood slides, admitted to hospital with malaria and then recruited to clinical research studies on the western border of Thailand between 1986 and 2002 were analysed. Patients were recruited at hospitals in Kanchanaburi (1986-1993; n = 571), Sangklaburi (1994-1995; $n=74)$, and Mae Sot $(1995-2002 ; n=343)$ and described in a series of papers [24]. All patients gave informed consent to participation and all studies were approved by the Ethics Committee of the Faculty of Tropical Medicine, Mahidol University and/or the Ethical and Scientific Review Subcommittee of the Ministry of Public Health, Government of Thailand.

\section{Clinical and laboratory assessment}

As patients were recruited to a variety of studies, clinical and laboratory evaluations varied. All patients had a full history and examination performed and haematocrit and parasitaemia determined. Thick and thin blood films were stained immediately with Field's stain and parasites counted and staged [7,8]. Admission blood samples for full blood count, serum sodium, potassium, creatinine, urea, total bilirubin, direct bilirubin, alkaline phosphatase, alanine transaminase (ALT), aspartate transaminase (AST), plasma lactate, glucose and, except in 1994, for plasma bicarbonate. Lumbar punctures were performed for the majority of patients with reduced GCS and cerebrospinal fluid cell, protein and glucose concentrations determined and Gram stains examined.

\section{Management}

The anti-malarial treatment regimens used in clinical studies in western Thailand changed over the 18 years as new anti-malarials were introduced and were:

1. Non-artemisinin-based parenteral treatment: Intravenous quinine dihydrochloride with or without a $20 \mathrm{mg}$ salt $/ \mathrm{kg}$ or $7 \mathrm{mg} / \mathrm{kg}$ loading dose followed by $10 \mathrm{mg} / \mathrm{kg}$ every $8 \mathrm{~h}$ followed by oral quinine salt $10 \mathrm{mg} / \mathrm{kg}$ every $8 \mathrm{~h}$, combined, when the patient was able to take oral medication, with oral quinine alone or with oral tetracycline, doxycycline, mefloquine (alone or combined with sulphadoxinepyrimethamine (SP)) or single dose primaquine to give a total treatment course of 7 days.

2. Non-artemisinin-based oral treatment: Oral quinine sulphate $10 \mathrm{mg}$ salt/kg every $8 \mathrm{~h}$ alone or with oral tetracycline, doxycycline, mefloquine (alone or combined with SP), single dose primaquine or proguanil to give a total treatment course of 7 days. Or oral mefloquine (alone or combined with SP) at $15 \mathrm{mg}$ base $/ \mathrm{kg}$ on the first day with or without 10 $\mathrm{mg} / \mathrm{kg}$ on the next day Or oral halofantrine $(8 \mathrm{mg} / \mathrm{kg}$ at $0,6,12 \mathrm{~h})$ with tetracycline plus or minus oral quinine

3. Artemisinin-based parenteral treatment: Intravenous artesunate (Guilin Pharmaceutical Factory No. 2, Guangxi, People's Republic of China); $2.4 \mathrm{mg} / \mathrm{kg}$ stat, $1.2 \mathrm{mg} / \mathrm{kg}$ at $12 \mathrm{~h}$ followed by $1.2 \mathrm{mg} / \mathrm{kg}$ every $24 \mathrm{~h})$ or intramuscular artemether $(3.2 \mathrm{mg} / \mathrm{kg}$ stat followed by $1.6 \mathrm{mg} / \mathrm{kg}$ every $24 \mathrm{~h}$ ) for 7 days alone or with oral doxycycline, tetracycline or mefloquine (alone or combined with SP) with or without primaquine. Sixty-nine patients were treated with iv artesunate combined with iv quinine followed by the above oral regime [25].

4. Artemisinin-based oral treatment: Oral artesunate or artemether $(4 \mathrm{mg} / \mathrm{kg}$ for 3 days or $2 \mathrm{mg} / \mathrm{kg}$ for 7 days) alone or with oral doxycycline, tetracycline or mefloquine (alone or combined with SP) with or without primaquine. Eighteen patients received oral dihydroartemisinin $(4 \mathrm{mg} / \mathrm{kg})$ in replacement for one artesunate daily dose [26].

Supportive treatment was in accordance with guidelines $[17,27]$. Facilities for urinary catheter and nasogastric tube placement, blood transfusion and lumbar puncture were available at Sangklaburi Hospital. In addition, mechanical ventilation, peritoneal dialysis and central venous access were available at Kanchanaburi and Mae Sot. 


\section{Statistical analysis and modelling}

Six main models have been used to define severe malaria in adults, those published by WHO and the AQ, MSA and CAM scores [16-23] (Additional file 1). The accuracy of the APACHE II score [28] could not be assessed as not all necessary laboratory variables were measured, and the definition of WHO 1986 was not evaluated [16]. Data were not collected specifically for this analysis and in order to examine the sensitivity and specificity of WHO 1990 and 2000 definitions [17,18], minor adaptations were made to allow assessment (Additional file 1, Tables 1 and 2). Where the WHO 2000 definition [18] does not include quantitative cut offs, the WHO 1990 [17] cut offs have been used. As blood $\mathrm{pH}$, and hence base deficit, data were not available, calculation of the CAM score was not possible and so the modified CAM scores, BCAM and RCAM were evaluated, using serum bicarbonate and respiratory rate, respectively, as surrogates of acidosis (Tables 1 and 2).

Statistical analyses were performed using Stata (v11.0; Stata Corporation, USA). All univariate comparisons between survivors and patients who died were performed using logistic regression and adjusted for study site. Ten sets of clinical and laboratory assay variables (Tables 1 and 2) were used to construct diagnostic rules to predict death. Logistic regression analysis with a stepwise forward variable selection procedure was employed to find independent predictors of death at $\mathrm{P}<0.055$, and $\mathrm{P} \geq 0.055$ for entry and removal, respectively. Fractional polynomials [29] were used to test for non-linear relationships between outcome variable and continuous covariates. All models were adjusted for artemisinin-based combination therapy (ACT) and study site. The identified model was rerun on the maximum available sample size and each of the variables not in the model were tested for inclusion using the Wald test. The predictive utility of each final model was assessed by receiver operating characteristic (ROC) curve analysis.

\section{Malaria prognostic index}

Variables selected into any final logistic regression model, based on published criteria (see above), were used to define the malaria prognostic index (MPI). Since for all logistic models (above), inclusion of study site as an independent variable did not improve the model nor change the co-efficients for other covariates significantly, it was not included in the development of the MPI. Each variable was categorized into four groups using rounded quartiles or commonly used cut offs (as for GCS). Univariate logistic models (with sets of corresponding binary variables) were fitted and categories with similar $(\mathrm{P}>0.05)$ odds ratios $(\mathrm{OR})$ were grouped together. Co-efficients of the final multivariate model were rounded to the nearest integer and used to calculate for each patient a linear combination of variables (i. e, sum of variables multiplied by the rounded co-efficients) - the MPI. The MPI was calculated for each patient and the ROC analysis used to evaluate its prognostic utility.

The predictive value of the MPI was further evaluated using cross validation [30] on a subset of data with no missing values for variables chosen to define MPI. Each observation in this subset was sequentially removed, logistic regression with stepwise variable selection was performed on the remaining $n-1$ observations using all categorized variables, and the final model was used to calculate sensitivity, specificity on $n-1$ observations and classification results for the one excluded observation. Co-efficients were rounded to integers and cut offs for the linear predictor between two and six were used. For each cut off, classification results for observations excluded from the subsequent models were used to calculate jacknife estimates of sensitivity and specificity.

\section{Results}

\section{Clinical presentation and outcome}

Of 988 hospitalized patients enrolled (Tables 3, 4, 5, 6, 7 and 8), two thirds were men and ages ranged from 15 to 74 years. The percentages with severe malaria, as defined by the WHO 1990, WHO 2000, adapted AQ, BCAM, RCAM and MSA scores [17,18,20-22], were widely spread at $61.2,78.1,41.7,24.8,22.7$ and $5.6 \%$, respectively (see Additional files 2, 3, and 4). The overall mortality was $7.8 \%$ (77/988). Using the WHO 1990, 2000 and adapted AQ definitions of severe malaria [17-20], the mortalities were $12.4 \%, 10 \%$ and $18.7 \%$, in these groups, respectively.

Mortality did not significantly differ between the three sites (9\% in Kanchanaburi, 3\% in Sangklaburi and 8\% in Mae Sot; $\mathrm{P}=0.20)$. Artemisinin derivatives were given to $7 \%$ of patients in Kanchanaburi, $77 \%$ in Sangklaburi and $83 \%$ in Mae Sot $(\mathrm{P}<0.001)$, reflecting temporal changes in treatment policy and study protocols. Patients in Kanchanaburi had received prior malarial treatment more frequently than at other sites and women there were more often pregnant. Patients in Mae Sot presented with lower coma scores (69\% with coma score of 15 compared to $>80 \%$ at the other sites and $20 \%$ with coma score $\leq 11$ compared to $9-10 \%$ in the other sites), higher parasitaemia, higher proportions of trophozoites on admission film and a greater likelihood of a palpable liver or spleen (Tables 3, 4, 5, and 6). However, other key variables such as haematocrit, bicarbonate and lactate were similar across all sites.

Forty-one admission variables were significantly associated $(\mathrm{P}<0.05)$ with death on bivariate analysis (Tables 7 and 8). Variables previously associated with mortality that were not significantly associated with death in our cohort were patient age, the number of days of illness before 
Table 3 Admission clinical history details for the three study sites

\begin{tabular}{|c|c|c|c|c|c|c|c|c|}
\hline \multirow[t]{2}{*}{ Variable } & \multicolumn{2}{|c|}{ All patients } & \multicolumn{2}{|c|}{ Kanchanaburi } & \multicolumn{2}{|c|}{ Sangklaburi } & \multicolumn{2}{|c|}{ Mae Sot } \\
\hline & $\mathbf{N}$ & Number (\%) & $\mathrm{N}$ & & $\mathrm{N}$ & & $\mathbf{N}$ & \\
\hline No. male & 982 & $654(67)$ & 569 & $371(65)$ & 74 & $42(57)$ & 339 & $241(71)$ \\
\hline Age/years & 979 & $27(15-74)$ & 564 & $26(15-72)$ & 73 & $25(15-57)$ & 342 & $28(15-74)$ \\
\hline Body weight/kg & 929 & $51(26-165)$ & 544 & $51(32-80)$ & 70 & $50(26-89)$ & 315 & $51(32-165)$ \\
\hline Height/cm & 441 & 160 (96-180) & 199 & 160 (96-78) & 2 & $154(145-163)$ & 240 & 160 (143-180) \\
\hline BMl kg/m $\mathrm{m}^{\mathrm{a}}$ & 440 & $33.5(32.8-34.2)$ & 198 & $33.0(32.3-33.8)$ & 2 & 42.1 & 240 & $33.8(32.7-34.8)$ \\
\hline Prior malaria & 780 & 445/335 (57) & 464 & 256/208 (55) & 52 & $26 / 26(100)$ & 264 & 163/101 (62) \\
\hline Prior malarial drug & 988 & $268 / 720(27)$ & 571 & 233/338 (41) & 74 & 6/68 (8) & 343 & 29/314 (9) \\
\hline No. days ill & 956 & $4(1-31)$ & 557 & $4(1-31)$ & 68 & $3(1-30)$ & 331 & $3(1-21)$ \\
\hline Females pregnant & 328 & $76 / 252(23)$ & 198 & $70 / 128(35)$ & 32 & $5 / 27(16)$ & 98 & $1 / 97(1)$ \\
\hline Headache & 921 & $843 / 78(92)$ & 520 & $484 / 36(93)$ & 72 & 66/6 (92) & 329 & 293/36 (89) \\
\hline Rigors & 875 & 454/421 (52) & 491 & 309/182 (63) & 60 & $15 / 45(25)$ & 324 & 130/194 (40) \\
\hline Vomiting & 906 & 5 17/389 (57) & 511 & 296/215 (58) & 69 & $37 / 32(54)$ & 326 & 184/142 (56) \\
\hline Diarrhoea & 850 & 160/690 (19) & 461 & 90/371 (24) & 65 & $8 / 57(12)$ & 324 & $62 / 262(19)$ \\
\hline Abdominal pain & 867 & 223/644 (26) & 478 & $129 / 349(27)$ & 65 & $7 / 58(11)$ & 324 & $87 / 237(27)$ \\
\hline Cough & 851 & 143/708 (17) & 470 & 99/371 (21) & 55 & $6 / 49(11)$ & 326 & $38 / 288(12)$ \\
\hline Seizures & 836 & 28/808 (3) & 456 & 9/447 (2) & 60 & $1 / 59$ (2) & 320 & 18/302 (6) \\
\hline
\end{tabular}

Number with/without symptom or sign or median (range) except ${ }^{\mathrm{a}}$ mean $\left(95 \% \mathrm{Cl}\right.$ ) and ${ }^{\mathrm{b}}$ geometric mean $(95 \% \mathrm{Cl})$. Percentages shown in brackets. Location of hospitals included: Kanchanaburi $\left(14.06^{\circ} \mathrm{N}, 99.50^{\circ} \mathrm{E}\right)$, Sangklaburi $\left.15.16^{\circ} \mathrm{N} 98.56^{\circ} \mathrm{E}\right)$, Mae Sot $\left(16.71^{\circ} \mathrm{N} 98.57^{\circ} \mathrm{E}\right)$.

Table 4 Admission clinical examination details for the three study sites

\begin{tabular}{|c|c|c|c|c|c|c|c|c|}
\hline \multirow{3}{*}{$\begin{array}{l}\text { Variable } \\
\text { Dehydration }\end{array}$} & \multicolumn{2}{|c|}{ All patients } & \multirow{2}{*}{\multicolumn{2}{|c|}{$\begin{array}{l}\text { Kanchanaburi } \\
\mathrm{N}\end{array}$}} & \multirow{2}{*}{\multicolumn{2}{|c|}{$\frac{\text { Sangklaburi }}{\mathrm{N}}$}} & \multirow{2}{*}{\multicolumn{2}{|c|}{$\frac{\text { Mae Sot }}{\mathrm{N}}$}} \\
\hline & \multirow{2}{*}{$\frac{\mathbf{N}}{781}$} & \multirow{2}{*}{$\begin{array}{l}\text { Number (\%) } \\
428 / 353(55)\end{array}$} & & & & & & \\
\hline & & & 424 & $182 / 242(43)$ & 41 & $13 / 28(32)$ & 316 & $233 / 83(74)$ \\
\hline Anaemia & 932 & 274/658 (29) & 529 & 161/368 (30) & 68 & 10/58 (15) & 335 & $103 / 232(31)$ \\
\hline Jaundice & 969 & 226/743 (23) & 561 & 137/424 (24) & 72 & $9 / 63(13)$ & 336 & $80 / 256(24)$ \\
\hline Temperature ${ }^{\circ} \mathrm{C}^{\mathrm{a}}$ & 963 & $38.5(38.4-38.6)$ & 552 & $38.4(38.3-38.5)$ & 74 & $38.5(38.3-38.8)$ & 337 & $38.7(38.5-38.8)$ \\
\hline Pulse/min ${ }^{a}$ & 927 & $101.0(99.9-102.2)$ & 519 & 97.4 (95.9-98.9) & 73 & $103.1(99.2-106.9)$ & 335 & $106.2(104.6-108.0)$ \\
\hline BP systolic $\mathrm{mmHg}^{\mathrm{a}}$ & 924 & $108.3(107.3-109.3)$ & 515 & $107.5(106.2-108.9)$ & 71 & $105.7(102.2-109.3)$ & 338 & $110.1(108.3-111.8)$ \\
\hline BP diastolic mmHg ${ }^{a}$ & 918 & $65.5(64.7-66.3)$ & 513 & $64.3(63.3-65.4)$ & 71 & $68.8(66.6-71.0)$ & 334 & $66.7(65.3-68.0)$ \\
\hline Respiratory rate/min ${ }^{b}$ & 909 & $24.3(23.8-24.7)$ & 508 & $22.8(22.2-23.3)$ & 70 & $28.1(26.6-29.8)$ & 331 & $25.9(25.2-26.6)$ \\
\hline Abdominal tenderness & 801 & $124 / 677(15)$ & 413 & $61 / 352(15)$ & 73 & $8 / 65(11)$ & 315 & $55 / 260(17)$ \\
\hline Liver palpable & 870 & 338/532 (39) & 500 & 183/317 (37) & 68 & $7 / 61(10)$ & 302 & 148/154 (49) \\
\hline Spleen palpable & 885 & 190/695 (22) & 506 & $94 / 412(19)$ & 71 & 0/71 (0) & 308 & $96 / 212(31)$ \\
\hline Cranial nerve abnormalities & 515 & $35 / 480(7)$ & 230 & 23/207 (10) & 15 & 2/13 (13) & 270 & $10 / 260(4)$ \\
\hline GCS Total & 944 & $15(3-15)$ & 529 & $15(3-15)$ & 73 & $15(3-15)$ & 342 & $15(3-15)$ \\
\hline GCS Eyes & 941 & $4(1-4)$ & 526 & $4(1-4)$ & 73 & $4(1-4)$ & 342 & $4(1-4)$ \\
\hline GCS Verbal & 939 & $5(1-5)$ & 526 & $5(1-5)$ & 73 & $5(1-5)$ & 342 & $5(1-5)$ \\
\hline GCS Motor & 941 & $6(1-6)$ & 526 & $6(1-6)$ & 73 & $6(1-6)$ & 342 & $6(1-6)$ \\
\hline Fundal haemorrhages & 443 & $11 / 432(3)$ & 202 & 6/196 (3) & 10 & $1 / 9(10)$ & 231 & $4 / 227(2)$ \\
\hline
\end{tabular}

Number with/without or median (range) except ${ }^{\mathrm{a}}$ mean $(95 \% \mathrm{Cl})$ and ${ }^{\mathrm{b}}$ geometric mean $(95 \% \mathrm{Cl})$. 
Table 5 Admission haematology laboratory details for the three study sites

\begin{tabular}{|c|c|c|c|c|c|c|c|c|}
\hline \multirow[t]{2}{*}{ Variable } & \multicolumn{2}{|c|}{ All patients } & \multicolumn{2}{|c|}{ Kanchanaburi } & \multicolumn{2}{|c|}{ Sangklaburi } & \multicolumn{2}{|c|}{ Mae Sot } \\
\hline & $\mathrm{N}$ & Number (\%) & $\mathrm{N}$ & & $\mathrm{N}$ & & $\mathrm{N}$ & \\
\hline Parasitaemia $/ \mu \mathrm{L}^{\mathrm{b}, \mathrm{c}}$ & 953 & $72,694(64.402-82,054)$ & 540 & $53,766(45,785-63,138)$ & 74 & $71,793(51,736-99,625)$ & 339 & $117,827(95,989-144,633)$ \\
\hline Rings \% & 874 & $97(1-100)$ & 486 & $97(6-100)$ & 66 & $98(42-100)$ & 322 & $95(1-100)$ \\
\hline Trophozoites \% & 874 & $3(0-99)$ & 486 & $2.5(0-94)$ & 66 & $2.0(0-58)$ & 323 & $4.0(0-99)$ \\
\hline Schizonts \% & 876 & $0(0-25)$ & 486 & $0(0-12)$ & 67 & $0(0-6)$ & 320 & $0(0-25)$ \\
\hline Pigmented stages $>10^{4} / \mathrm{L}$ & 868 & 274/594 (32) & 482 & $121 / 361(25)$ & 66 & $13 / 53(20)$ & 322 & 140/180 (43) \\
\hline Trophozoites and schizonts\% & 874 & $4(0-99)$ & 486 & $3(0-94)$ & 66 & $2(0-58)$ & 321 & $5(0-99)$ \\
\hline Modal Stage \% & 833 & 55 (19-99) & 446 & 56 (19-99) & 66 & $70(30-96)$ & 339 & $53(23-97)$ \\
\hline Haematocrit \% & 962 & $37(6-56)$ & 549 & $37(8-56)$ & 74 & $38(22-50)$ & 285 & $38(6-54)$ \\
\hline Haemoglobin $\mathrm{g} / \mathrm{dL}^{\mathrm{a}}$ & 761 & $11.3(11.1-11.5)$ & 476 & $10.9(10.7-11.1)$ & 0 & - & 314 & $12.0(11.6-12.3)$ \\
\hline White cells $\times 10^{9} / \mathrm{L}$ & 889 & $6.8(1.5-67.0)$ & 519 & $6.6(1.8-67)$ & 56 & $5.7(1.5-25)$ & 292 & $7.1(1.9-54)$ \\
\hline Neutrophils \% & 863 & $75(12-97)$ & 507 & $73(31-97)$ & 64 & $74(12-96)$ & 281 & $77(41-96)$ \\
\hline Platelets $\times 10^{9} / \mathrm{L}$ & 527 & $74(0.5-404)$ & 246 & $105(16-384)$ & 0 & - & 329 & $45(1-404)$ \\
\hline
\end{tabular}

${ }^{c}$ number of asexual parasites/1000 erythrocytes on thin film $\mathrm{x}$ haematocrit $\% \times 125.6$ or number of asexual parasites/200 white cells on thick film assuming white cell count $8 \times 10^{9} / \mathrm{L}$.

admission, and plasma glucose. Although there was no apparent overall relationship between mortality and age, mortality was higher with increasing age for those treated with quinine (OR (95\% CI) 1.029 (1.006-1.051) P = 0.012), but not for those treated with artemisinins (OR 0.953 (0.9041.005) $\mathrm{P}=0.078)$.

\section{Prognostic value of parasite staging}

Staging of parasite development on peripheral blood smears provides prognostic information additional to the parasite count itself; the median percentage of ring stages amongst those who survived was $98 \%$ and amongst those who died $48 \%$ ( $\mathrm{P}=0.0001)$. The percentage of the modal

Table 6 Admission biochemistry laboratory variables for the three study sites

\begin{tabular}{|c|c|c|c|c|c|c|c|c|}
\hline \multirow[t]{2}{*}{ Variable } & \multicolumn{2}{|c|}{ All patients } & \multicolumn{2}{|c|}{ Kanchanaburi } & \multicolumn{2}{|c|}{ Sangklaburi } & \multicolumn{2}{|c|}{ Mae Sot } \\
\hline & $\bar{N}$ & value & $\bar{N}$ & value & $\mathrm{N}$ & value & $\bar{N}$ & value \\
\hline Sodium mmol/L'd S & 764 & $135.4(93.8-155.0)$ & 419 & $137.8(94-155)$ & 16 & 136.5 (130-141) & 328 & $133(111-146)$ \\
\hline Potassium mmol/L, e s & 761 & $3.81(1.2-7.51)$ & 417 & $3.8(1.2-7.5)$ & 16 & $3.70(3.3-4.3)$ & 331 & $3.9(1.8-7.1)$ \\
\hline Creatinine $\mu \mathrm{mol} / \mathrm{L}^{\mathrm{fs}}$ & 880 & $105.6(35.2-1056)$ & 475 & $114(35-1056)$ & 74 & 97 (66-202) & 334 & $106(39-836)$ \\
\hline Urea mmol/L g s & 892 & $19.5(3.5-226.5)$ & 484 & $6.5(1.5-56)$ & 74 & $6.7(2.5-18.7)$ & 334 & $7.7(1.3-81)$ \\
\hline Urea $\mathrm{mmol} / \mathrm{L}:$ creatinine $\mu \mathrm{mol} / \mathrm{L}$ ratio & 879 & $0.070(0.005-0.256)$ & 474 & $0.060(0.005-0.244)$ & 74 & $0.067(0.022-0.014)$ & 331 & $0.078(0.021-0.026)$ \\
\hline Total bilirubin $\mu \mathrm{mol} / \mathrm{L}$ b,h, s & 859 & $28.9(26.8-31.0)$ & 456 & $23.6(21.1-26.4)$ & 73 & $29.4(24.5-35.2)$ & 330 & $38.0(34.6-41.8)$ \\
\hline Direct bilirubin $\mu \mathrm{mol} / L^{1, S}$ & 847 & $8.5(0.3-488.6)$ & 451 & $8.5(0.3-487)$ & 72 & $6.1(1.5-152)$ & 324 & $9.9(1.0-389)$ \\
\hline Alkaline phosphatase IU/L ${ }^{b, j, s}$ & 844 & $43.2(41.1-45.3)$ & 442 & $31.5(29.8-33.3)$ & 73 & $34.9(31.9-38.2)$ & 329 & $69.2(64.2-74.6)$ \\
\hline AST IU/L ${ }^{k, S}$ & 858 & $41(0.4-1795)$ & 455 & $33(5-210)$ & 73 & $43(0.4-183)$ & 330 & $52(10-1795)$ \\
\hline$A L T \| / L^{b, l, s}$ & 856 & $20.7(19.5-22.0)$ & 456 & $19.2(17.6-21.1)$ & 73 & $24.5(21.3-28.2)$ & 327 & $22.2(20.4-24.1)$ \\
\hline Albumin $\mathrm{g} / \mathrm{L}^{\mathrm{a}, \mathrm{m}, \mathrm{s}}$ & 862 & $33.7(33.2-34.2)$ & 459 & $31.9(31.2-32.7)$ & 73 & $38.6(37.0-40.1)$ & 330 & $35.0(34.3-35.7)$ \\
\hline Calcium (uncorrected) mmol/L a,n, s & 397 & $2.06(1.47-2.56)$ & 0 & - & 73 & $2.12(2.09-2.15)$ & 324 & $2.05(2.04-2.07)$ \\
\hline Phosphate $\mathrm{mmol} / \mathrm{L}^{\mathrm{b}, \mathrm{o}, \mathrm{s}}$ & 291 & $0.82(0.32-2.65)$ & 0 & - & 73 & $1.19(0.32-1.87)$ & 218 & $0.81(0.32-2.65)$ \\
\hline Bicarbonate $\mathrm{mmol} / \mathrm{L}^{\mathrm{a}, \mathrm{p}, \mathrm{s}}$ & 687 & $20.5(20.2-20.8)$ & 348 & $21.0(20.5-21.4)$ & 16 & $20.4(18.9-22.0)$ & 323 & $20.0(19.4-20.5)$ \\
\hline Chloride $\mathrm{mmol} / \mathrm{L}^{\mathrm{a}, \mathrm{q}, \mathrm{s}}$ & 752 & $102.2(101.7-102.7)$ & 407 & $103.7(103.0-104.3)$ & 16 & $103.3(101.2-105.3)$ & 329 & 100.2 (99.6-100.8) \\
\hline Glucose $\mathrm{mmol} / \mathrm{L}^{\mathrm{r}, \mathrm{P}}$ & 846 & $6.4(0.7-37.5)$ & 447 & $6.2(1.5-38)$ & 70 & $6.2(3.5-25)$ & 329 & $7.3(0.7-31.1)$ \\
\hline Lactate $\mathrm{mmol} / \mathrm{L}^{\mathrm{s}, \mathrm{P}}$ & 805 & $2.7(0.3-27.6)$ & 414 & $2.7(0.4-28)$ & 74 & $2.2(0.9-16)$ & 317 & $2.8(0.3-22)$ \\
\hline
\end{tabular}

Number with/without or median (range) except ${ }^{\mathrm{a}}$ mean $(95 \% \mathrm{Cl})$ and ${ }^{\mathrm{b}}$ geometric mean $(95 \% \mathrm{Cl})$.

${ }^{\mathrm{d}}$ normal range $135-145 \mathrm{mmol} / \mathrm{L} ;{ }^{\mathrm{e}}$ normal range $3.5-5.5 \mathrm{mmol} / \mathrm{L} ;{ }^{\mathrm{f}}$ normal range $70-150 \mu \mathrm{mol} / \mathrm{L} ;{ }^{\mathrm{g}}$ normal range $2.5-6.7 \mathrm{mmol} / \mathrm{L} ;{ }^{\mathrm{h}}$ normal range $5.1-17 \mu \mathrm{mol} / \mathrm{L}$;

${ }^{\prime}$ normal range $1.7-5.1 \mathrm{mmol} / \mathrm{L} ;{ }^{j}$ normal range $30-300 \mathrm{IU} / \mathrm{L} ;{ }^{k}$ normal range $5-35 \mathrm{lU} / \mathrm{L} ;{ }^{1}$ normal range $5-35 \mathrm{IU} / \mathrm{L} ;{ }^{\mathrm{m}}$ normal range $35-50 \mathrm{~g} / \mathrm{L} ;{ }^{\mathrm{n}}$ normal range 2.2-2.6 mmol $/ \mathrm{L}_{i}{ }^{\circ}$ normal range $1.0-1.4 \mathrm{mmol} / \mathrm{L}_{i}{ }^{\mathrm{p}}$ normal range $21-28 \mathrm{mmol} / \mathrm{L} ;{ }^{\mathrm{q}}$ normal range $95-105 \mathrm{mmol} / \mathrm{L} ;{ }^{\mathrm{r}}$ normal range $3.5-5.5 \mathrm{mmol} / \mathrm{L} ;{ }^{\mathrm{s}}$ normal $<4 \mathrm{mmol} / \mathrm{L}$. 
Table 7 Admission clinical variables for those who died and survived

\begin{tabular}{|c|c|c|c|c|c|c|c|c|c|c|}
\hline \multirow{3}{*}{$\begin{array}{l}\text { Variable } \\
\text { No. male }\end{array}$} & \multicolumn{3}{|c|}{ All patients } & \multicolumn{3}{|c|}{ Survived } & \multicolumn{3}{|c|}{ Died } & \multirow[t]{2}{*}{$\mathbf{P}$} \\
\hline & \multicolumn{2}{|l|}{$\mathrm{N}$} & \multirow{2}{*}{$\begin{array}{l}\% \\
67\end{array}$} & \multicolumn{2}{|l|}{$\mathrm{N}$} & \multirow{2}{*}{$\begin{array}{l}\% \\
66\end{array}$} & \multicolumn{2}{|l|}{$\mathrm{N}$} & \multirow{2}{*}{$\begin{array}{l}\% \\
74\end{array}$} & \\
\hline & 982 & 654 & & 906 & 598 & & 76 & 56 & & 0.197 \\
\hline Age/years & 979 & $27(15-74)$ & - & 905 & $27(15-74)$ & - & 74 & $26(15-67)$ & - & 0.410 \\
\hline Body weight/kg & 929 & $51(26-165)$ & - & 861 & $51(26-165)$ & - & 68 & $50(40-75)$ & - & 0.715 \\
\hline Prior malaria & 780 & $445 / 335$ & 57 & 748 & $438 / 310$ & 59 & 32 & $7 / 25$ & 22 & $<0.001$ \\
\hline Prior malarial drug & 988 & $268 / 720$ & 27 & 911 & $242 / 669$ & 27 & 77 & $26 / 51$ & 34 & 0.310 \\
\hline No. days ill & 956 & $4(1-31)$ & - & 886 & $3(1-31)$ & - & 70 & $4(1-20)$ & - & 0.836 \\
\hline No. females pregnant & 328 & $76 / 252$ & 23 & 308 & $75 / 233$ & 24 & 20 & $1 / 19$ & 5 & 0.049 \\
\hline Headache & 921 & $843 / 78$ & 92 & 866 & $794 / 72$ & 94 & 55 & $49 / 6$ & 89 & 0.511 \\
\hline Rigors & 875 & $454 / 421$ & 52 & 821 & $428 / 393$ & 52 & 54 & $26 / 28$ & 48 & 0.522 \\
\hline Vomiting & 906 & $517 / 389$ & 57 & 855 & $484 / 371$ & 56 & 51 & $33 / 18$ & 65 & 0.268 \\
\hline Diarrheoa & 850 & $160 / 690$ & 19 & 805 & $152 / 653$ & 19 & 45 & $8 / 37$ & 18 & 0.805 \\
\hline Abdominal pain & 867 & $223 / 644$ & 26 & 823 & $215 / 608$ & 26 & 44 & $8 / 36$ & 18 & 0.200 \\
\hline Cough & 851 & $143 / 708$ & 17 & 806 & $136 / 670$ & 17 & 45 & $7 / 38$ & 16 & 0.897 \\
\hline Seizures & 836 & $28 / 808$ & 3 & 786 & $18 / 768$ & 2 & 50 & $10 / 40$ & 20 & $<0.001$ \\
\hline Dehydration & 781 & $428 / 353$ & 55 & 734 & $395 / 339$ & 54 & 47 & $33 / 14$ & 70 & 0.063 \\
\hline Anaemia & 932 & $274 / 658$ & 29 & 862 & $236 / 626$ & 27 & 70 & $38 / 32$ & 54 & $<0.001$ \\
\hline Jaundice & 969 & $226 / 743$ & 23 & 892 & $175 / 717$ & 20 & 77 & $51 / 26$ & 66 & $<0.001$ \\
\hline Temperature ${ }^{\circ} \mathrm{C}^{\mathrm{a}}$ & 963 & $38.5(38.4-38.6)$ & - & 892 & $38.6(38.5-38.6)$ & - & 71 & $38.0(37.7-38.3)$ & - & $<0.001$ \\
\hline Pulse/min ${ }^{a}$ & 927 & $101.0(99.9-102.2)$ & - & 856 & $100.1(99.0-101.2)$ & - & 71 & $113.3(108.6-117.9)$ & - & $<0.001$ \\
\hline BP systolic $\mathrm{mmHg}{ }^{a}$ & 924 & $108.3(107.3-109.3)$ & - & 851 & 107.9 (106.9-108.9) & - & 73 & $113.5(108.4-118.5)$ & - & 0.004 \\
\hline BP diastolic $\mathrm{mmHg}^{\mathrm{a}}$ & 918 & $65.5(64.7-66.3)$ & - & 846 & $65.4(64.6-66.2)$ & - & 72 & $66.8(63.6-70.0)$ & - & 0.247 \\
\hline Respiratory rate/min ${ }^{b}$ & 909 & $24.3(23.8-24.7)$ & - & 837 & $23.9(23.5-24.3)$ & - & 71 & $29.2(26.9-31.6)$ & - & $<0.001$ \\
\hline Abdominal tenderness & 801 & $124 / 677$ & 15 & 743 & $116 / 627$ & 16 & 58 & $8 / 50$ & 14 & 0.689 \\
\hline Liver palpable & 870 & $338 / 532$ & 39 & 800 & $299 / 501$ & 38 & 70 & $39 / 31$ & 56 & 0.005 \\
\hline Spleen palpable & 885 & 190/695 & 22 & 818 & $172 / 646$ & 21 & 67 & $18 / 49$ & 27 & 0.308 \\
\hline Cranial nerve abnormalities & 515 & $35 / 480$ & 7 & 467 & $20 / 447$ & 4 & 48 & $15 / 33$ & 31 & $<0.001$ \\
\hline GCS Total & 944 & $15(3-15)$ & - & 882 & $15(3-15)$ & - & 62 & $9(3-15)$ & - & $<0.001$ \\
\hline GCS Eyes & 941 & $4(1-4)$ & - & 881 & $4(1-4)$ & - & 60 & $4(1-4)$ & - & $<0.001$ \\
\hline GCS Verbal & 939 & $5(1-5)$ & - & 881 & $5(1-5)$ & - & 58 & $1(1-5)$ & - & $<0.001$ \\
\hline GCS Motor & 941 & $6(1-6)$ & - & 881 & $6(1-6)$ & - & 60 & $5(1-6)$ & - & $<0.001$ \\
\hline Fundal haemorrhages & 443 & $11 / 432$ & 3 & 389 & $4 / 385$ & 1 & 54 & $7 / 47$ & 13 & $<0.001$ \\
\hline
\end{tabular}

Number with/without or median (range) except ${ }^{a}$ mean $(95 \% \mathrm{Cl})$ and ${ }^{\mathrm{b}}$ geometric mean $(95 \% \mathrm{Cl})$. Footnotes as for Table 3.

parasite stage was significantly lower amongst those who died than in those who survived $(P=0.0002)$, suggesting that lower circulating parasite stage synchronicity was associated with death. Using the cut offs of Silamut and White of pigmented stages $>10^{4} / \mu \mathrm{L}$ or a parasitaemia of $>5 \times 10^{5} / \mu \mathrm{L}$ [7], the sensitivity and specificity for predicting death were $84 \%$ and $67 \%$, respectively.

\section{Therapeutic responses}

The median (range) coma recovery time and time to death were $24(1-188) \mathrm{h}(\mathrm{N}=46)$ and $44(2-641) \mathrm{h}(\mathrm{N}=76)$ respectively (Table 9). Artemisinin derivative-based therapy was given to $378 / 958$ (39\%) of patients starting in 1993, and artesunate or artemether was given to $317 / 759$ (42\%) of those administered a parenteral anti-malarial. A significantly higher proportion of patients had severe disease (as defined using adapted AQ criteria) in the artemisinin derivative group (46\%) than the nonartemisinin group $(40 \%)(\mathrm{P}<0.001)$. However, the mortality in those given parenteral artesunate or artemether $(16 / 317,5 \%)$ was lower than those given parenteral quinine $(59 / 442,13 \%)(\mathrm{P}<0.001)$. No patient without severe disease who received oral anti-malarial treatment 
Table 8 Admission laboratory variables for those who died and survived

\begin{tabular}{|c|c|c|c|c|c|c|c|}
\hline \multirow[t]{2}{*}{ Variable } & \multicolumn{2}{|c|}{ All patients } & \multicolumn{2}{|c|}{ Survived } & \multicolumn{2}{|c|}{ Died } & \multirow[t]{2}{*}{$\mathbf{P}$} \\
\hline & $\mathrm{N}$ & value & $\mathrm{N}$ & value & $\mathrm{N}$ & value & \\
\hline Admission parasitaemia $\mu \mathrm{L}^{\mathrm{b}, \mathrm{c}}$ & 953 & $72,694(64,402-82,054)$ & 883 & $68,850(60,837-77,917)$ & 70 & $144,012(86,460-239,876)$ & 0.002 \\
\hline Rings\% & 874 & $97(1-100)$ & 811 & $98(1-100)$ & 63 & $48(1-100)$ & $<0.001$ \\
\hline Trophozoites\% & 874 & $3(0-99)$ & 811 & $2(0-99)$ & 63 & $52(0-99)$ & $<0.001$ \\
\hline Schizonts\% & 876 & $0(0-25)$ & 813 & $0(0-6)$ & 63 & $0(0-25)$ & $<0.001$ \\
\hline Pigmented stages $>10^{4} / \mathrm{L}$ & 868 & $274 / 594(32 \%)$ & 805 & $227 / 578(28 \%)$ & 63 & $47 / 16(75 \%)$ & $<0.001$ \\
\hline Trophozoites and schizonts \% & 874 & $4(0-99)$ & 811 & $2(0-99)$ & 63 & $52(0-99)$ & $<0.001$ \\
\hline Modal Stage \% & 833 & 55 (19-99) & 779 & $56(23-99)$ & 54 & 47 (19-94) & 0.001 \\
\hline Haematocrit \% & 962 & $37(6-56)$ & 888 & $37(6-56)$ & 74 & $33(12-50)$ & $<0.001$ \\
\hline Haemoglobin $\mathrm{g} / \mathrm{dL}^{\mathrm{a}}$ & 761 & $11.3(11.1-11.5)$ & 704 & $11.4(11.2-11.6)$ & 57 & $10.1(9.5-10.8)$ & $<0.001$ \\
\hline White cells $\times 10^{9} / \mathrm{L}$ & 889 & $6.8(1.5-67.0)$ & 818 & $6.6(1.5-67)$ & 71 & $11.2(2.4-63)$ & $<0.001$ \\
\hline Neutrophils\% & 863 & $75(12-97)$ & 796 & $75(12-97)$ & 67 & $72(36-95)$ & 0.101 \\
\hline Platelets $10^{9} / \mathrm{L}$ & 527 & $74(0.5-404)$ & 491 & $75(0.5-404)$ & 36 & $50(4-188)$ & 0.001 \\
\hline Sodium mmol/L, $d, s$ & 764 & $135.4(93.8-155.0)$ & 698 & $135.3(100.4-155.0)$ & 66 & $136.7(93.8-153.0)$ & 0.618 \\
\hline Potassium mmol/L' e, S & 761 & $3.81(1.2-7.51)$ & 696 & $3.80(2.0-7.51)$ & 65 & $4.50(1.2-7.1)$ & $<0.001$ \\
\hline Creatinine $\mu \mathrm{mol} / \mathrm{L}^{\mathrm{f}, \mathrm{S}}$ & 880 & $105.6(35.2-1056)$ & 810 & $105.6(35.2-1056)$ & 70 & $240(46.6-880)$ & $<0.001$ \\
\hline Urea $\mathrm{mmol} / \mathrm{L}^{\mathrm{g}} \mathrm{S}$ & 892 & $19.5(3.5-226.5)$ & 821 & $18.7(3.5-138)$ & 71 & $58.2(10.7-227)$ & $<0.001$ \\
\hline Urea $\mathrm{mmol} / \mathrm{L}:$ creatinine $\mu \mathrm{mol} / \mathrm{L}$ ratio & 879 & $0.070(0.005-0.256)$ & 809 & $0.065(0.005-0.257)$ & 70 & $0.078(0.030-0.260)$ & $<0.001$ \\
\hline Total bilirubin $\mu \mathrm{mol} / \mathrm{L}^{\mathrm{b}, \mathrm{h}}$ & 859 & $28.9(26.8-31.0)$ & 797 & $26.4(24.6-28.4)$ & 62 & $90.6(69.6-118.1)$ & $<0.001$ \\
\hline Direct bilirubin $\mu \mathrm{mol} / \mathrm{L}^{i}$ & 847 & $8.5(0.3-488.6)$ & 787 & $8.3(0.3-489)$ & 60 & $40.3(1.4-389)$ & $<0.001$ \\
\hline Alkaline phosphatase IU/L ${ }^{b, j}$ & 844 & $43.2(41.1-45.3)$ & 781 & $42.1(40.0-44.2)$ & 63 & $60.7(50.5-72.8)$ & $<0.001$ \\
\hline AST IU/L ${ }^{k}$ & 858 & $41.0(0.4-1795)$ & 796 & $40(0.4-1795)$ & 62 & $95(22-1200)$ & 0.001 \\
\hline$A L T I U / L{ }^{b, l}$ & 856 & $20.7(19.5-22.0)$ & 794 & $19.7(18.5-20.9)$ & 62 & $41.3(33.6-50.8)$ & $<0.001$ \\
\hline Albumin $g / L^{a, m}$ & 862 & $33.7(33.2-34.2)$ & 798 & $33.9(33.4-34.4)$ & 64 & $30.6(28.5-32.7)$ & 0.001 \\
\hline Calcium (uncorrected) $\mathrm{mmol} / \mathrm{L}^{\mathrm{a}, \mathrm{n}}$ & 397 & $2.06(1.47-2.56)$ & 369 & $2.06(1.52-2.56)$ & 28 & $2.06(1.47-2.40)$ & 0.385 \\
\hline Phosphate $\mathrm{mmol} / \mathrm{L}^{\mathrm{b}, \mathrm{o}}$ & 291 & $0.82(0.32-2.65)$ & 272 & $0.81(0.32-2.10)$ & 19 & $1.23(0.48-2.65)$ & $<0.001$ \\
\hline Serum bicarbonate $\mathrm{mmol} / \mathrm{L}^{\mathrm{a}, \mathrm{p}}$ & 687 & $20.5(20.2-20.8)$ & 629 & $21.0(20.7-21.3)$ & 58 & $14.7(13.1-16.3)$ & $<0.001$ \\
\hline Serum chloride $\mathrm{mmol} / \mathrm{L}^{\mathrm{a}, \mathrm{q}}$ & 752 & $102.2(101.7-102.7)$ & 688 & $102.3(101.9-102.7)$ & 64 & $100.6(98.0-103.2)$ & 0.023 \\
\hline Plasma glucose $\mathrm{mmol} / \mathrm{L}^{\mathrm{r}}$ & 846 & $6.4(0.7-37.5)$ & 778 & $6.4(0.7-38)$ & 68 & $6.8(2.2-18.5)$ & 0.139 \\
\hline Plasma lactate $\mathrm{mmol} / \mathrm{L}^{\mathrm{s}}$ & 805 & $2.7(0.3-27.6)$ & 741 & $2.5(0.3-22)$ & 64 & $8.5(1.9-28)$ & $<0.001$ \\
\hline
\end{tabular}

Number with/without or median (range) except ${ }^{\mathrm{a}}$ mean $(95 \% \mathrm{Cl})$ and ${ }^{\mathrm{b}}$ geometric mean $(95 \% \mathrm{Cl})$. Footnotes as for Tables 3, 5 and 6.

died. The post-admission development of complications such as oliguria, seizures or pulmonary oedema and the use of ventilation, lumbar puncture, transfusion and inotropes, were all associated with death (Table 10). Mortality decreased with time, from $9 \%$ in $1986-1992$ to $7 \%$ in $1993-1998$ and $6 \%$ in $1999-2002(\mathrm{OR}=0.943$ (0.896-0.994), $\mathrm{P}=0.030)$. There was increasing use of artemisinin derivatives over the same periods $(0 \%, 72 \%$ and $93 \%$ of patients, respectively) and the corresponding percentages of parenteral treatments that were with intravenous/intramuscular artemisinin derivative were $0 \%, 67 \%$ and $93 \%$, respectively. After adjusting for treatment type and study site, there was no trend in mortality over time $(\mathrm{OR}=0.961(0.847-1.091), \mathrm{P}=0.538)$.

\section{Relationships between clinical syndromes}

Of 111 patients with cerebral malaria (GCS < 11), 97 had evaluable data. Of these, 68 (70\%) had plasma lactate $>4 \mathrm{mmol} / \mathrm{L}$ and/or serum bicarbonate $<15 \mathrm{mmol} / \mathrm{L}$ and/ or serum creatinine $>264 \mu \mathrm{mol} / \mathrm{L}$, and $36(53 \%)$ of these died compared with three (10\%) among 29 cerebral malaria patients without these abnormalities $(\mathrm{P}=0.001)$. Among cerebral malaria patients, mortality did not significantly differ between those with and without jaundice (total serum bilirubin $>50 \mu \mathrm{mol} / \mathrm{L}$ ): $42 \%$ (23/55) versus $34 \%(15 / 44) \quad(\mathrm{P}=0.547)$. Mortality was higher in patients with renal impairment; 82\% (18/22) in those with a serum creatinine $>264 \mu \mathrm{mol} / \mathrm{L}$ compared with $28 \%(23 / 83)$ in those with lower levels $(\mathrm{P}<0.001)$. 
Table 9 Anti-malarial drug treatment and outcome

\begin{tabular}{|c|c|c|c|c|c|c|}
\hline \multirow[t]{2}{*}{ Variable } & \multicolumn{2}{|l|}{ All } & \multicolumn{2}{|l|}{ Alive } & \multicolumn{2}{|l|}{ Died } \\
\hline & $+/-$ & $\%$ & $+/-$ & $\%$ & $+/-$ & $\%$ \\
\hline Non-artemisinin/artemisinin derivative therapy & $580 / 378$ & 60.5 & $521 / 362$ & 59.0 & $59 / 16$ & 78.7 \\
\hline $\begin{array}{l}\text { Parenteral quinine/artemisinin derivative. Patients given both parenteral } \\
\text { quinine and artemisinin derivatives excluded }\end{array}$ & $430 / 276$ & 60.9 & $371 / 261$ & 58.7 & $59 / 15$ & 79.7 \\
\hline Oral quinine/artemisinin derivative and no parenteral anti-malarial & $93 / 60$ & 60.8 & $93 / 60$ & 60.8 & $0 / 0$ & - \\
\hline Number given/not given antibiotic ${ }^{a}$ & $124 / 818$ & 13.2 & $94 / 778$ & 10.8 & $30 / 40$ & 42.9 \\
\hline No. given/not given inotrope & $65 / 872$ & 6.9 & $13 / 856$ & 1.5 & $52 / 16$ & 76.5 \\
\hline No. given/not given anti-epileptic drug & $97 / 843$ & 10.3 & $59 / 812$ & 6.8 & $38 / 31$ & 55.1 \\
\hline No. given/not given nasogastric tube & $65 / 859$ & 7.0 & $36 / 828$ & 4.2 & $29 / 31$ & 48.3 \\
\hline No. given/not given urinary catheter & $207 / 728$ & 22.1 & $142 / 725$ & 16.4 & $65 / 3$ & 95.6 \\
\hline No. given/not given blood transfusion & $160 / 828$ & 16.2 & $132 / 779$ & 14.5 & $28 / 49$ & 36.4 \\
\hline No. given/not given dialysis & $31 / 898$ & 3.3 & $7 / 854$ & 0.8 & $24 / 44$ & 77.4 \\
\hline No. given/not given ventilation & $85 / 845$ & 9.1 & $25 / 839$ & 2.9 & $60 / 6$ & 90.9 \\
\hline No. given/not given central venous access line & $99 / 840$ & 10.5 & $45 / 825$ & 5.2 & $54 / 15$ & 78.3 \\
\hline No. given/not given lumbar puncture & $90 / 849$ & 9.6 & $56 / 813$ & 6.4 & $34 / 36$ & 48.6 \\
\hline No. developing/not developing pulmonary oedema & $14 / 958$ & 1.4 & $7 / 888$ & 0.8 & $7 / 70$ & 9.1 \\
\hline No. developing/not developing oliguria & $94 / 843$ & 10.0 & $43 / 826$ & 5.0 & $51 / 17$ & 75.0 \\
\hline No. developing/not developing seizures & $39 / 909$ & 4.1 & $20 / 852$ & 2.3 & $19 / 57$ & 25.0 \\
\hline No. of nights in hospital ${ }^{\mathrm{b}}$ & $4(1-34)$ & - & $4(1-34)$ & - & $2(1-27)$ & - \\
\hline Coma Recovery Time/ $\mathrm{h}^{\mathrm{b}}$ & $24(1-188)$ & - & - & - & - & - \\
\hline Time to death $/ h^{b}$ & - & - & - & - & $44(2-641)$ & - \\
\hline
\end{tabular}

${ }^{a}$ excluding doxycycline and tetracycline given for malaria. ${ }^{\mathrm{b}}$ Median (range). All comparisons between alive and dead were statistically significant $(\mathrm{P}<0.001$, adjusted for study site).

\section{Multiple logistic regression analysis of variables associated with death}

All final models were adjusted for treatment with ACT and study site. No interactions between covariates and treatment were significant in any of the models and study site was not significant $(P>0.05)$. Tables 10 and 11 list variables included in each of the eight final models, all having a maximum of eight variables, consistent with recommendations [31].

Considering the WHO- and AQ-based models, the WHO 1990, WHO 2000, Adapted AQ and Adapted AQ + Pigmented stages, variable sets gave the best predictive power and the areas under the ROC curves (AUROCCs) were not significantly different (Tables 10 and 11, Additional files 2, 3, and 4). Models derived from bedside or bedside + simple laboratory covariates had significantly smaller AUROCCs, when compared to the Adapted AQ model using the common data set $(P=0.008$ and 0.016 , respectively). Several variables appeared in all four 'best' models and had similar effects across models, such as GCS (OR 0.67-0.73 for increase by one point), serum bicarbonate (OR 0.84-0.86 for increase of $1 \mathrm{mmol} / \mathrm{L}$ ), plasma lactate (OR 1.20-1.34 for increase of $1 \mathrm{mmol} / \mathrm{L}$ ), parasitaemia (OR 1.90-4.06 for ten-fold increase) and serum creatinine (OR 1.005-1.006 for increase of $1 \mu \mathrm{mol} / \mathrm{L}$ ).
The BCAM and RCAM scores could be calculated for 662 and 873 patients, respectively, and mortality rose with increasing score for both (Table 12). Of 498 patients with a low BCAM $(<2)$ score, three $(0.6 \%)$ died (PPV (95\%CI) for survival 99.4 (98.2-99.8)\%), and of the 675 patients with low RCAM $(<2)$ score, 11 (1.6\%) died (PPV for survival 98.4 (97.1-99.1)\%). The BCAM score AUROCC as a predictor for mortality was 0.907 (0.861-0.953) and that for the RCAM score $0.86(0.81-0.91)(\mathrm{P}=0.050)$. A score $<2$ was an optimal cut-off value in this data set for both BCAM and RCAM, with sensitivity and specificity of $93.8 \%$ and $80.6 \%$ for BCAM and $81 \%$ and $81 \%$ for RCAM.

For the MSA score [22] (Additional file 1) using admission variables plus the presence or absence of mechanical ventilation during admission where such ventilation was available (all sites except Sangklaburi) for 635 patients, $516(81.3 \%)$ had an MSA score of 0 . Mortality was $2 / 539$ (0.4\%) for MSA $0-2,10 / 45$ (22.2\%) for MSA 3-4, 5/12 (41.7\%) for MSA $5-6$ and $24 / 39$ (61.5\%) for MSA $\geq 7$. If $\geq 5$ is taken as the cut off, the PPV for survival was 97.9 (96.4-98.8)\%. Among patients who had MSA and BCAM scores calculated $(n=527)$ the AUROCC for the MSA score in predicting death was 0.97 (0.95-0.98), which was significantly better than that for the BCAM score 0.90 
Table 10 Summary of multiple logistic regression models for outcome for Bedside and WHO models

\begin{tabular}{|c|c|c|c|c|c|}
\hline Covariate & OR & $95 \% \mathrm{Cl}$ & & $P$ value & AUROCC $95 \% \mathrm{Cl}$ \\
\hline Bedside & & & & & $0.93(0.90-0.96)$ \\
\hline Anaemia & 2.510 & 1.173 & 5.373 & 0.018 & \\
\hline Jaundice & 3.235 & 1.534 & 6.823 & 0.002 & \\
\hline Temperature ${ }^{\circ} \mathrm{C}$ & 0.673 & 0.490 & 0.926 & 0.015 & \\
\hline Pulse/min & 1.031 & 1.007 & 1.056 & 0.010 & \\
\hline Respiratory rate/min & 1.061 & 1.010 & 1.115 & 0.018 & \\
\hline GCS & 0.717 & 0.654 & 0.785 & $<0.001$ & \\
\hline Treatment with ACT & 0.387 & 0.134 & 1.118 & 0.080 & \\
\hline Bedside + Simple Lab & & & & & $0.96(0.93-0.98)$ \\
\hline Temperature ${ }^{\circ} \mathrm{C}$ & 0.579 & 0.405 & 0.829 & 0.003 & \\
\hline Respiratory rate/min & 1.081 & 1.027 & 1.139 & 0.003 & \\
\hline $\log _{10}$ parasitaemia & 8.606 & 3.525 & 21.011 & $<0.001$ & \\
\hline GCS & 0.663 & 0.589 & 0.745 & $<0.001$ & \\
\hline$\%$ Trophozoites and schizonts & 1.029 & 1.014 & 1.045 & $<0.001$ & \\
\hline Treatment with $\mathrm{ACT}$ & 0.253 & 0.078 & 0.825 & 0.023 & \\
\hline WHO (1990) & & & & & $0.95(0.90-1.00)$ \\
\hline GCS & 0.673 & 0.590 & 0.767 & $<0.001$ & \\
\hline Serum creatinine $\mu \mathrm{mol} / \mathrm{L}$ & 1.005 & 1.001 & 1.009 & 0.007 & \\
\hline Serum bicarbonate $\mathrm{mmol} / \mathrm{L}$ & 0.844 & 0.763 & 0.934 & 0.001 & \\
\hline Serum total bilirubin $\mu \mathrm{mol} / \mathrm{L}$ & 1.006 & 1.001 & 1.010 & 0.011 & \\
\hline $\log _{10}$ parasitaemia & 1.902 & 1.060 & 3.410 & 0.031 & \\
\hline Treatment with ACT & 0.838 & 0.224 & 3.142 & 0.794 & \\
\hline WHO (2000) and Adapted AQ ${ }^{a}$ & & & & & $0.97(0.96-0.99)$ \\
\hline GCS & 0.673 & 0.582 & 0.778 & $<0.001$ & \\
\hline Serum bicarbonate $\mathrm{mmol} / \mathrm{L}$ & 0.856 & 0.747 & 0.980 & 0.024 & \\
\hline Serum lactate $\mathrm{mmol} / \mathrm{L}$ & 1.197 & 1.052 & 1.362 & 0.006 & \\
\hline $\log _{10}$ parasitaemia & 2.460 & 1.168 & 5.182 & 0.018 & \\
\hline Serum creatinine $\mu \mathrm{mol} / \mathrm{L}$ & 1.006 & 1.002 & 1.010 & 0.003 & \\
\hline Treatment with ACT & 1.000 & 0.220 & 4.555 & 1.000 & \\
\hline
\end{tabular}

All estimates are adjusted for treatment with ACT and study site. ${ }^{a}$ the same variables were identified from the WHO (2000) and Adapted AQ models.

(0.84-0.96) $(\mathrm{P}=0.007)$. The optimal cut off for the data presented here appeared to be $<3$ rather than $<5$ as reported [22]; with a cut off of $<3$ the PPV was 99.6 (98.7-100.0)\%, sensitivity $95.1 \%$ and specificity $90.4 \%$.

Considering all ten models, those based on the MSA score and the Adapted AQ with pigmented parasites had the best predictive power, but AUROCCs for models based on WHO criteria were not significantly lower. Simple rules for classification of severity as a MPI (Table 13, Figure 1), gave sensitivity of $100 \%$ and specificity of $82 \%$ with co-efficient of 3 rounded to the nearest integer, and sensitivity of $93 \%$ and specificity of $92 \%$ for a cut off of 4 . When compared to the best performing models, based on MSA and Adaptive AQ + Pigmented stages, MPI showed equally good predictive power:
AUROCC $=0.96(0.95-0.98)$ for MPI rounded to the nearest integer, and 0.97 (0.96-0.99) for MPI rounded to the nearest 0.5 compared to 0.98 (0.96-0.99) for MSA and 0.97 (0.94-0.99) for AQ dataset, based on 450 patients who could be evaluated in all four models. In cross-validation, in all runs, the best sensitivity and specificity were obtained for cut offs between 3 and 4 (Figure 2). For a cut off of 3, the jacknife sensitivity and specificity were $97.1 \%$ and $87.1 \%$; for a cut off of 3.5 they were $97.1 \%$ and $87.1 \%$; and for a cut off of 4 they were $74 \%$ and $94 \%$.

\section{Discussion}

The prognosis of severe falciparum malaria has improved markedly since the introduction of parenteral artesunate 
Table 11 Summary of multiple logistic regression models for outcome for Adapted AQ + pigmented stages, BCAM and RCAM models

\begin{tabular}{|c|c|c|c|c|c|}
\hline Covariate & OR & $95 \% \mathrm{Cl}$ & & $P$ value & AUROCC $95 \% \mathrm{Cl}$ \\
\hline Adapted $A Q+$ pigmented stages & & & & & $0.97(0.96-0.99)$ \\
\hline GCS & 0.728 & 0.641 & 0.828 & $<0.001$ & \\
\hline Plasma lactate $\mathrm{mmol} / \mathrm{L}$ & 1.341 & 1.190 & 1.510 & $<0.001$ & \\
\hline Serum creatinine $\mu \mathrm{mol} / \mathrm{L}$ & 1.006 & 1.003 & 1.009 & $<0.001$ & \\
\hline$\%$ Trophozoites \& schizonts & 1.026 & 1.009 & 1.043 & 0.003 & \\
\hline $\log _{10}$ parasitaemia & 4.064 & 1.506 & 10.969 & 0.006 & \\
\hline Treatment with ACT & 0.438 & 0.103 & 1.869 & 0.265 & \\
\hline BCAM & & & & & $0.92(0.87-0.97)$ \\
\hline GCS/15 & 0.718 & 0.652 & 0.791 & $<0.001$ & \\
\hline Serum bicarbonate $\mathrm{mmol} / \mathrm{L}$ & 0.780 & 0.717 & 0.849 & $<0.001$ & \\
\hline Treatment with $\mathrm{ACT}$ & 0.718 & 0.230 & 2.237 & 0.568 & \\
\hline RCAM & & & & & $0.88(0.84-0.93)$ \\
\hline GCS/15 & 0.680 & 0.627 & 0.736 & $<0.001$ & \\
\hline Respiratory rate/min & 1.097 & 1.055 & 1.141 & $<0.001$ & \\
\hline Treatment with ACT & 0.329 & 0.126 & 0.857 & 0.023 & \\
\hline
\end{tabular}

All estimates are adjusted for treatment with ACT and study site.

[21,32]. This large series describing patients with falciparum malaria admitted to hospitals in western Thailand spanned the transition from quinine to artemisinins, and although the data were not from a randomized comparison, mortality was substantially lower in patients who received artemisinin derivatives. Despite the effect of changes in anti-malarial therapy, prognostic indices based on WHO 2000 definitions, and other simpler indices based on fewer variables, provided clinically useful predictions of outcome in Asian adults with severe malaria.

Models using variable sets based on WHO 2000 and Adapted $\mathrm{AQ}$ with pigmented stages gave the best prediction of mortality, and were comparable to the results based on the MSA score using a smaller sample. Very similar results were also obtained with the MPI based on the most commonly selected variables, GCS, plasma lactate, parasitaemia, serum bilirubin and percentage of pigmented parasites. This will need to be evaluated in independent series of adults with falciparum malaria in similar settings of unstable malaria transmission. This score suffers from the disadvantage, unlike the RCAM score, that determination of 4/5 variables (all except GCS) requires skilled technicians and equipment/consumables and quality assurance that are seldom available where severe malaria is common.

There are at least four limitations of this analysis: specifically, not all patients admitted to the study hospitals were recruited, recruitment criteria varied, there are missing values, and a variety of different doctors reviewed the patients with consequent variability in the nature of both clinical assessment and inpatient management. However, any such differences are likely to result in false negative, rather than false positive associations. The MSA score differs from the others scores discussed, as it is not strictly an admission predictive prognostic score, including mechanical ventilation during hospitalization. Some potential prognostic factors such as haemoglobinuria and abnormal bleeding were too infrequent to allow reliable conclusions to be drawn. Serum bilirubin and plasma lactate were not measured in the South East Asian Quinine Artesunate Malaria Trial (SEAQUAMAT) and therefore the MPI could not be calculated for this dataset [21].

Table 12 Mortality among patients by BCAM and RCAM scores

\begin{tabular}{|c|c|c|c|c|c|c|}
\hline Score, Outcome & 0 & 1 & 2 & 3 & 4 & Total \\
\hline \multicolumn{7}{|l|}{ BCAM } \\
\hline Died/total (\%) & $1 / 124(0.8)$ & $2 / 374(0.5)$ & $12 / 79(15.2)$ & $16 / 60(26.7)$ & 17/25 (68.0) & 48/662 (7.3) \\
\hline \multicolumn{7}{|l|}{ RCAM } \\
\hline Died/total (\%) & 0/97 (0) & $11 / 578(1.9)$ & 12/108 (11.1) & $25 / 74(33.8)$ & $11 / 16(68.8)$ & $59 / 873(6.8$ \\
\hline
\end{tabular}


Table 13 Prognostic index for severe malaria

\begin{tabular}{|c|c|c|c|}
\hline \multirow[t]{2}{*}{$N=668 / n=43$} & \multicolumn{3}{|c|}{ Co-efficient } \\
\hline & & MPI A & MPI B \\
\hline GCS $<5$ & 4.318 & 4 & 4.5 \\
\hline GCS 5-11 & 1.543 & 2 & 1.5 \\
\hline 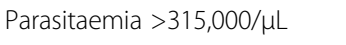 & 1.238 & 1 & 1 \\
\hline Plasma lactate $>5 \mathrm{mmol} / \mathrm{L}$ & 2.267 & 2 & 2.5 \\
\hline Serum bilirubin $>58 \mu \mathrm{mol} / \mathrm{L}^{1}$ & 1.191 & 1 & 1 \\
\hline Pigmented parasites > 20\% & 1.673 & 2 & 1.5 \\
\hline Treatment with ACT & -1.280 & -1 & -1.5 \\
\hline AUROCC & 0.97 & 0.97 & 0.97 \\
\hline Cut off ${ }^{2}$ & & 3 & 3 \\
\hline Sensitivity & & $100 \%$ & $100 \%$ \\
\hline Specificity & & $82 \%$ & $88 \%$ \\
\hline Cut off $^{3}$ & & 4 & 3.5 \\
\hline Sensitivity & & $93 \%$ & $95 \%$ \\
\hline Specificity & & $92 \%$ & $91 \%$ \\
\hline
\end{tabular}

MPI A = co-efficients rounded to the nearest integer with cut offs of 3. MPI B $=$ co-efficients rounded to the nearest 0.5 with cut offs of 3 .

1 if not available; creatinine $>132 \mu \mathrm{mol} / \mathrm{L}$ can be used instead. and cut off of 3 would give sensitivity and specificity of $98 \%$. and $82 \%$ for MPI A and $96 \%$ and $88 \%$ for MPI B.

${ }^{2}$ which gives the highest specificity for $100 \%$ sensitivity.

${ }^{3}$ which gives the "optimal" sensitivity and specificity.

This study differs from that of Hanson [23], which only included patients classified as having severe malaria and was designed to determine which subset of patients could safely be managed without ICU referral. This is reflected in the relatively low mortality in the series described here, as severe malaria was not necessarily a criterion for recruitment. The broader patient population included may well explain the inclusion of parasitaemia as predictive of death in this series and not in that of Hanson [23]. As this series includes many patients

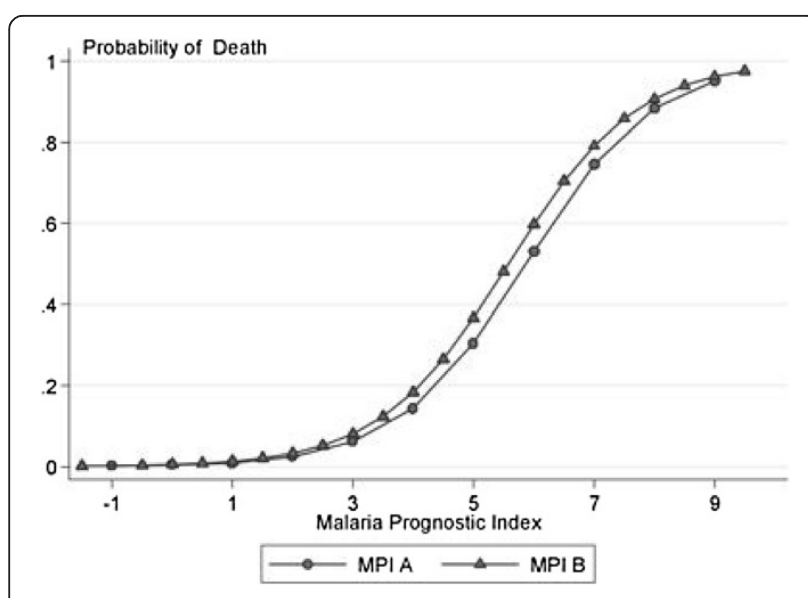

Figure 1 Relationship between malaria prognostic index and mortality. Malaria prognostic indices MPI A and MPI B are defined in Table 13.

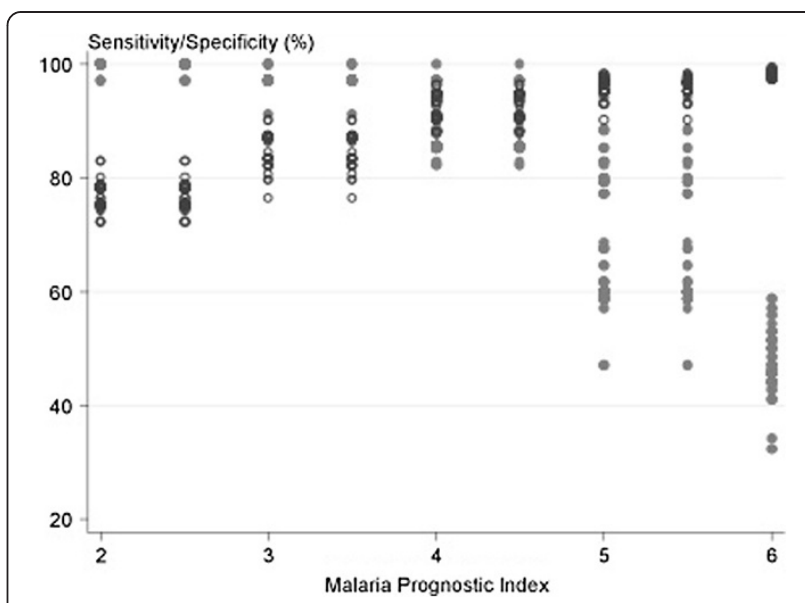

Figure 2 Sensitivity (filled circle) and specificity (empty circle) for different cut offs of malaria prognostic index in cross validation.

without severe disease, the specificity of variables in predicting death may be higher than in series including patients with pre-selected severe disease. However, in busy clinical practice a tool that could define unselected patients admitted to hospital as at risk of death would be valuable.

The wide variation in mortality estimates in this dataset among those with severe malaria (10-18\%) and indeed the wide range of estimates of severe disease (6-60\%), depending on which definition is used, illustrates the importance of definitions in comparisons between studies, in metaanalyses, and in understanding the host and geographical variability in the presentation and outcome of severe malaria. Various terms, such as 'uncomplicated' and 'mild', are used to refer to malaria that is not severe. Severe disease is also equated with 'complicated' malaria. To avoid confusion, terminology should be standardized with 'severe' malaria referring to malaria with clinical and/or laboratory features suggesting a clinically significant risk of death (e.g. $>5 \%$ ) despite anti-malarial treatment, and 'uncomplicated' as those without such prognostic features and that the terms 'complicated' and 'mild' are not used.

The present study suggests that, if laboratory tests are available, the history of the illness and the physical examination, apart from GCS and respiratory rate, are relatively unimportant in assessing prognosis in a population of malaria patients. Unlike in other series [13], increased age was not associated with death, except for those treated with quinine. However, as this series did not include children and a smaller percentage (7.7\%) were $>50$ years old, the age range was narrower.

In this series, the mortality of cerebral malaria was increased three-fold if concurrent acidosis and/or renal failure were present and these factors are crucial in predicting death. The ease of identifying patients with 
cerebral malaria may have made study of other complications less of a focus and these data suggest that more research on the pathophysiology and treatment of acidosis and renal dysfunction would provide information that would improve management and outcome [12,14,32]. The use of relatively inexpensive plasma lactate portable meters may assist in the triage of patients in tropical hospitals without access to biochemical analyzers, and interventions directed at acidosis and renal impairment might reduce mortality. Although capital costs for veno-venous haemofiltration are relatively high, and intensive care support is required, running costs in comparison to peritoneal dialysis are low [33]. In areas of the Asiatic tropics with good communications but low health expenditure, regional centres for the management of severe malaria through haemofiltration may assist in lowering mortality.

\section{Additional files}

Additional file 1: Severe malaria definitions.
Additional file 2: World Health Organization (1990) criteria for
severe malaria and outcome.
Additional file 3: World Health Organization (2000) criteria for
severe malaria and outcome.
Additional file 4: Adapted 'AQ' criteria, from Hien et al. $[19,20]$, and
outcome.

\section{Competing interests}

The authors declare that they have no competing interest.

\section{Authors' contributions}

$\mathrm{PN}$ and $\mathrm{KS}$ analysed the data and wrote the first draft of the manuscript. PN, $A D, K S, W C, S K, T M E D, Y S, B A, S P, R R, J H, N D, N W$ looked after the patients and revised the manuscript. All authors read and approved the final manuscript.

\section{Acknowledgements}

We thank all the patients and hospital staff at Kanchanaburi, Sangklaburi and Mae Sot, all the staff of the Mahidol University Oxford Tropical Medicine Research Unit, especially Khun Patchari Prakongpan and the Faculty of Tropical Medicine, Mahidol University. We are extremely grateful to the numerous people of many nationalities who have contributed to the care of these patients and the associated clinical research in Thailand over many years. TMED is supported by a National Health and Medical Research Council Practitioner Fellowship. This study was supported by the Wellcome TrustMahidol University-Oxford Tropical Medicine Research Programme, funded by the Wellcome Trust of Great Britain.

\section{Author details \\ ${ }^{1}$ Lao-Oxford-Mahosot Hospital-Wellcome Trust Research Unit, Microbiology Laboratory, Mahosot Hospital, Vientiane, Lao PDR. ${ }^{2}$ Faculty of Tropical Medicine, Mahidol University, Bangkok, Thailand. ${ }^{3}$ Centre for Tropical Medicine, Churchill Hospital, Nuffield Department of Medicine, University of Oxford, Oxford OX3 7LJ, UK. “Division of Clinical Sciences, St George's Hospital, University of London, London, UK. ${ }^{5}$ School of Medicine \& Pharmacology, University of Western Australia, Freemantle, Australia. ${ }^{6}$ Department of Medicine, Siriraj Hospital, Bangkok, Thailand. ${ }^{7}$ Mae Sot Hospital, Mae Sot, Tak Province, Thailand. ${ }^{8}$ Menzies School of Health Research, Darwin, Australia.}

Received: 17 February 2013 Accepted: 23 June 2013

Published: 8 July 2013

\section{References}

1. World Health Organization: Global Malaria Report 2011. Geneva: World Health Organization; 2011. http://www.who.int/entity/malaria/ world_malaria_report_2011/9789241564403_eng.pdf.

2. Molyneux ME, Taylor TE, Wirima JJ, Borgstein A: Clinical features and prognostic indicators in paediatric cerebral malaria: a study of 131 comatose Malawian children. Q J Med 1989, 71:441-459.

3. Walker O, Salako LA, Sowunmi A, Thomas JO, Sodeine O, Bondi FS: Prognostic risk factors and post-mortem findings in cerebral malaria in children. Trans R Soc Trop Med Hyg 1992, 86:491-493.

4. Marsh K, Foster D, Waruiru C, Mwangi I, Winstanley M, Marsh V, Newton C, Winstanley P, Warn P, Peshu N, Pasvol G, Snow R: Indicators of life-threatening malaria in African children. New Eng J Med 1995, 332:1399-1404.

5. Jaffar S, Van Hensbroek MB, Palmer A, Schdieer G, Greenwood B: Predictors of fatal outcome following childhood cerebral malaria. Am J Trop Med Hyg 1997, 57:20-24.

6. Von Seidlein L, Olaosebikan R, Hendriksen IC, Lee SJ, Adedoyin OT, Agbenyega T, Nguah SB, Bojang K, Deen JL, Evans J, Fanello Cl, Gomes E, Pedro AJ, Kahabuka C, Karema C, Kivaya E, Maitland K, Mokuolu OA, Mtove G, Mwanga-Amumpaire J, Nadjm B, Nansumba M, Ngum WP, Onyamboko MA, Reyburn H, Sakulthaew T, Silamut K, Tshefu AK, Umulisa N, Gesase S, Day NP, White NJ, Dondorp AM: Predicting the clinical outcome of severe falciparum malaria in African children: findings from a large randomized trial. Clin Infect Dis 2012, 54:1080-1090.

7. Silamut K, White NJ: Relation of the stage of parasite development in the peripheral blood to prognosis in severe falciparum malaria. Trans $R$ Soc Trop Med Hyg 1993, 87:436-443.

8. Phu NH, Day N, Diep PT, Ferguson DJP, White NJ: Intraleucocytic malaria pigment and prognosis in severe malaria. Trans R Soc Trop Med Hyg 1995, 89:200-204

9. Trang TTM, Phu NH, Vinh H, Hien TT, Cuong BM, Chau TT, Mai NT, Waller DJ, White NJ: Acute renal failure in patients with severe falciparum malaria. Clin Infec Dis 1992, 15:874-880.

10. White NJ, Warrell DA, Chanthavanich P, Looareesuwan S, Warrell MJ, Krishna S, Williamson DH, Turner RC: Severe hypoglycemia and hyperinsulinemia in falciparum malaria. N Engl J Med 1983, 309:61-66.

11. Warrell DA, Looareesuwan S, Warrell MJ, Kasemsarn P, Intaraprasert R, Bunnag D, Harinasuta T: Dexamethasone proves deleterious in cerebral malaria. New Eng J Med 1982, 306:313-319.

12. Day NPJ, Phu NH, Mai NTH, Chau TT, Loc PP, Chuong LV, Sinh DX, Holloway P, Hien TT, White NJ: The pathophysiological and prognostic significance of acidosis in severe adult malaria. Crit Care Med 2000, 28:1833-1840.

13. Dondorp AM, Lee SJ, Faiz MA, Mishra S, Price R, Tjitra E, Than M, Htut Y, Mohanty S, Yunus EB, Rahman R, Nosten F, Anstey NM, Day NP, White NJ: The relationship between age and the manifestations of and mortality associated with severe malaria. Clin Infect Dis 2008, 47:151-157.

14. Dondorp AM, Chau TTH, Phu NH, Mai NT, Loc PP, Chuong LV, Sinh DX Taylor A, Hien TT, White NJ, Day NP: Unidentified acids of strong predictive value in severe malaria. Crit Care Med 2004, 32:1683-1688.

15. Bejon P, Berkley JA, Mwangi T, Ogada E, Mwangi I, Maitland K, Williams T, Scott JA, English M, Lowe BS, Peshu N, Newton CR, Marsh K: Defining childhood severe falciparum malaria for intervention studies. PLOS Med 2007, 4:e251.

16. World Health Organization: Severe and complicated malaria. Trans $R$ Soc Trop Med Hyg 1986, 80: . Supplement: 1-50.

17. World Health Organization: Severe and complicated malaria. Trans $R$ Soc Trop Med Hyg 1990, 84(S2):1-65.

18. World Health Organization: Severe and complicated malaria. Trans $R$ SoC Trop Med Hyg 2000, 94. Supplement 1:1-90.

19. Hien TT, Day NPJ, Nguyen HP, Nguyen THM, Tran THC, Pham PL, Dinh XS, Ly VC, Ha V, Waller D, Peto TEA, White NJ: A controlled trial of artemether or quinine in Vietnamese adults with severe falciparum malaria. N Engl J Med 1996, 335:76-83.

20. Newton PN, Angus BJ, Chierakul W, Dondorp A, Ruangveerayuth R, Silamut K, Teerapong P, Suputtamongkol Y, Looareesuwan S, White NJ: A randomised comparison of artesunate and quinine in the treatment of severe falciparum malaria. Clin Inf Dis 2003, 37:7-16.

21. Dondorp A, Nosten F, Stepniewska K, Day N, White N: South East Asian Quinine Artesunate Malaria Trial (SEAQUAMAT) group: Artesunate versus quinine for the treatment of severe falciparum malaria: a randomised trial. Lancet 2005, 366:717-725. 
22. Mishra SK, Panigrahi P, Mishra R, Mohanty S: Prediction of outcome in adults with severe falciparum malaria: a new scoring system. Malar J 2007, 6:24.

23. Hanson J, Lee SJ, Mohanty S, Faiz MA, Anstey NM, Charunwatthana P, Yunus EB, Mishra SK, Tjitra E, Price RN, Rahman R, Nosten F, Htut Y, Hoque G, Hong Chau TT, Hoan Phu N, Hien TT, White NJ, Day NP, Dondorp AM: A simple score to predict the outcome of severe malaria in adults. Clin Infect Dis 2010, 50:679-685.

24. White NJ: Twenty years of the Wellcome-Mahidol University, Oxford Tropical Medicine Research Program. Southeast Asian J Trop Med Pub Hlth 1999, 30:216-219.

25. Newton PN, Chierakul W, Ruangveerayuth R, Silamut K, Teerapong P, Krudsood S, Looareesuwan S, White NJ: A comparison of artesunate alone with combined artesunate and quinine in the parenteral treatment of acute falciparum malaria. Trans Roy Soc Trop Med Hyg. 2001, 95:519-523.

26. Newton PN, Van Vugt M, Teja-Isavadharm P, Keeratithakul D, Rasameesoroj M, Teerapong P, Ruangveerayuth R, Slight T, Nosten F, Suputtamongkol Y, Looareesuwan S, White NJ: A comparison of oral artesunate and dihydroartemisinin antimalarial bioavailability in acute falciparum malaria. Antimicrob Agents Chemother 2002, 46:125-127.

27. White NJ: The treatment of malaria. N Engl J Med 1996, 335:800-806.

28. Wilairatana P, Looareesuwan S: APACHE II scoring for predicting outcome in cerebral malaria. J Trop Med Hyg 1995, 98:256-260.

29. Royston P, Altman DG: Regression using fractional polynomials of continuous covariates: Parsimonious parametric modeling (with discussion). Appl Stats 1994, 43:429-467.

30. Lachenbruch PA, Mickey MR: Estimation of error rates in discriminant analysis. Technometrics 1969, 10:1-11.

31. Peduzzi P, Concato J, Feinstein AR, Holford TR: Importance of events per independent variable in proportional hazards regression analysis II. Accuracy and precision of regression estimates. J Clin Epidemiol 1995, 48:1503-1510.

32. Dondorp AM, Fanello Cl, Hendriksen IC, Gomes E, Seni A, Chhaganlal KD, Bojang K, Olaosebikan R, Anunobi N, Maitland K, Kivaya E, Agbenyega T, Nguah SB, Evans J, Gesase S, Kahabuka C, Mtove G, Nadjm B, Deen J, Mwanga-Amumpaire J, Nansumba M, Karema C, Umulisa N, Uwimana A, Mokuolu OA, Adedoyin OT, Johnson WB, Tshefu AK, Onyamboko MA, Sakulthaew T, Ngum WP, Silamut K, Stepniewska K, Woodrow CJ, Bethell D, Wills B, Oneko M, Peto TE, Von Seidlein L, Day NP, White NJ, AQUAMAT group: Artesunate versus quinine in the treatment of severe falciparum malaria in African children (AQUAMAT): an open-label, randomised trial. Lancet 2010, 376:1647-1657.

33. Phu NH, Hien TT, Mai NTH, Chau TT, Chuong LV, Loc PP, Winearls C, Farrar J, White N, Day N: Hemofiltration and peritoneal dialysis in infection associated acute renal failure in Vietnam. New Eng J Med 2002, 347:895-902.

doi:10.1186/1475-2875-12-229

Cite this article as: Newton et al:: Prognostic indicators in adults

hospitalized with falciparum malaria in Western Thailand. Malaria Journal 2013 12:229.

\section{Submit your next manuscript to BioMed Central and take full advantage of:}

- Convenient online submission

- Thorough peer review

- No space constraints or color figure charges

- Immediate publication on acceptance

- Inclusion in PubMed, CAS, Scopus and Google Scholar

- Research which is freely available for redistribution 